\title{
Are our ideas about Dst correct?
}

\author{
A. Grafe \\ GeoForschungsZentrum Potsdam, Telegrafenberg, D-14473 Potsdam, Germany
}

Received: 10 February 1998 / Revised: 11 May 1998 / Accepted: 2 June 1998

\begin{abstract}
The idea of two separate storm time ring currents, a symmetric and an asymmetric one has accepted since the 1960s. The existence of a symmetric equatorial ring current was concluded from Dst. However, the asymmetric development of the low-latitude geomagnetic disturbance field during storms lead to the assumption of the real existence of an asymmetric ring current. I think it is time to inquire whether this conception is correct. Thus, I have investigated the development of the low-latitude geomagnetic field during all the magnetic local times under disturbed and quiet conditions. The storm on February 6-9, 1986 and a statistical analysis of many storms has shown that the asymmetry does not vanish during the storm recovery phase. The ratio between the recovery phase asymmetry and the main phase asymmetry is low only for powerful storms. Storms of moderate intensity show the opposite. The global picture of the field evolution of the February storm shows clear differences at different local times. For instance the main phase and recovery phase start time does not coincide with Dst. Also the ring current decay is not the same at different local times. Therefore, Dst gives an incorrect picture of the field development. Moreover, asymmetry does not disappear during international quiet days as the investigation of the lowlatitude geomagnetic field shows. Considering all these observations, I think we must revise our ideas about the ring current. In my opinion only one ring current exists and this is an asymmetric one. This asymmetry increases during storms and develops rather fast to more or less symmetric conditions. However, in no case is it justified to conclude from Dst that a symmetric ring current exists.
\end{abstract}

Key words. Magnetospheric physics (current systems; magnetospheric configuration and dynamics; storms and substorms).

\section{Introduction}

The idea of two separate storm time ring currents, a symmetric and an asymmetric one has been accepted since the 1960s. The evidence for existence of a symmetric equatorial ring current was concluded from Dst. The birth of the term storm time variation goes back to the work of Moos (1910). Later Chapman (1919) called this variation $D s t$. The first activity indices of Dst were developed by Vestine et al. (1947) long before Sugiura (1964) published his Dst indices which have been used since then for the description of the storm time variation. As a result of the calculation of Dst as the first term of the Fourier analysis of the geomagnetic field $D s t$ is only a function of the time and the dipole latitude and not of the dipole longitude. It is always important to establish again that Dst is only a mathematical parameter obtained by analysing the geomagnetic field observations and nothing more. Its physical meaning is not obvious. The result of this analysis does say anything about the external sources of Dst. However, in the past some magnetospheric sources were proposed which can generate a symmetric magnetic field on the Earth's surface. Such sources are the magnetospheric current systems e.g. Chapman-Ferrarocurrent, symmetric equatorial ring current, field aligned currents, ionospheric currents, and tail current (Yacob, 1966, Campbell, 1973; Sun et al., 1984; Fuller-Rowell et al., 1994, Campbell, 1996, 1997). Supported by the discovery of the Van Allen radiation belts and other satellite observations (Frank, 1967) it is today generally accepted that $D s t$ is mainly caused by a symmetric ring current. This is in contrast to a present increasing of discussion about the influence of the tail current on Dst (Alexeev et al., 1996). However, Dst is only one component of the observed ground-based geomagnetic field. In principle the geomagnetic field is asymmetric at all latitudes on Earth. Chapman (1919) called this longitudinal inequality, the asymmetric deviations of the 
disturbance field from $D s t$, the $D S$ variation. More recently Akasofu and Chapman (1964) supposed an asymmetric ion charged particle distribution in the ring current region as the cause of the $D S$ variation in low latitudes. They assumed a separate flowing partial ring current which was confirmed by satellite observations discussed by Cahill (1966). Thereby, the idea of two independently occurring ring currents the symmetric and the asymmetric one was born. Later many papers were published about a symmetric and an asymmetric ring current (Cummings 1966; Grafe, 1968; Kamide and Fukushima, 1971; Crooker and McPherron, 1972; Fukushima and Kamide, 1973; Siscoe and Crooker, 1974; Williams, 1981). Lui et al. (1987) investigating direct satellite observations could show that the ring current has a fine structure pointing to many separated small asymmetric ring currents.

Today the existence of a symmetric and an asymmetric ring current is a fixed part of our knowledge about the processes in the inner magnetosphere. However, do we have clear evidence that our conception is right? I think we have no evidence. Why do we conclude the existence of a symmetric ring current from the calculated Dst variation? I could never understand this conclusion. This conclusion was drawn before knowledge about the population of the ring current particles in the inner magnetosphere was available. This population is more or less asymmetric as satellite observations show. Doubtless the asymmetry of the low-latitude magnetic field increases strongly during the storm main phase and decreases during the storm recovery phase. However, there are two fundamental questions:

1. How does the asymmetry of the low-latitude geomagnetic field develop during the magnetic storm phases and how is it related to the magnetic storm intensity?

2. Is the low-latitude geomagnetic field also asymmetric during quiet conditions? In the following investigation I want to try to answer these questions.

\section{Asymmetry of the low-latitude geomagnetic field during storm main and recovery phase}

The great geomagnetic storm on February 06-09, 1986

Magnetic field data of nine low-latitude geomagnetic observatories were used to estimate Dst and the asymmetry index ASY (Kawasaki and Akasofu, 1971). The position of observatories are shown in Table 1. The geomagnetic latitude of these observatories is about from 20 to $35^{\circ}$. The mean of the geomagnetic latitude of these observatories is $28.5^{\circ}$. The deviation of latitude for each station from this mean value gives a certain inaccuracy of the estimation of $D s t$ and $A S Y$. However, it is more important to have a sufficient longitudinal separation of the observatories. This requirement is fullfilled as Table 1 shows. The horizontal components of the geomagnetic disturbance field ( $S q$ eliminated) were converted into the geomagnetic oriented field
Table 1. Positions of observatories

\begin{tabular}{lllllc}
\hline Observatories & \multicolumn{2}{l}{ Geographic } & & \multicolumn{2}{c}{ Geomagnetic } \\
\cline { 2 - 3 } \cline { 5 - 6 } & latitude & longitude & & latitude & longitude \\
\hline San Juan & 18.1 & 293.9 & & 29.4 & 4.6 \\
M'Bour & 14.4 & 343.0 & & 20.9 & 56.4 \\
Tenerife & 28.5 & 343.7 & & 34.6 & 60.0 \\
Pendeli & 38.0 & 23.9 & & 36.3 & 102.8 \\
Kakioka & 36.2 & 140.2 & & 26.3 & 207.3 \\
Hatizyo & 33.1 & 139.8 & & 23.2 & 207.3 \\
Memambetsu & 43.9 & 144.2 & & 34.3 & 209.7 \\
Honolulu & 21.3 & 202.0 & & 21.4 & 268.0 \\
Teoloyucan & 19.8 & 260.8 & & 29.5 & 328.6 \\
\hline
\end{tabular}

components for all stations. The result of this extensive analysis is shown in Fig. 1. It is important to emphasize that $|D s t|$ reaches here only a value $<300 \mathrm{nT}$ whereas the Dst index of Sugiura (1964) reaches $-312 \mathrm{nT}$. This effect is caused by the fact that the calculated $D s t$ is valid for low latitudes, but not for the geomagnetic equator. This storm is characterized by a very long main phase of about two days as well as by a complex development. At least three steps of increasing $|D s t|$ could be registrated considering the main phase. The last one is the largest. The asymmetry of the disturbance field is strongly variable during the whole main phase. The intensity of $A S Y$ is similar to Dst during the last two steps. At the beginning of the recovery phase $A S Y$ decreases very quickly. This agrees with the opinion of Akasofu and Chapman (1972a). The method described by Grafe et al.

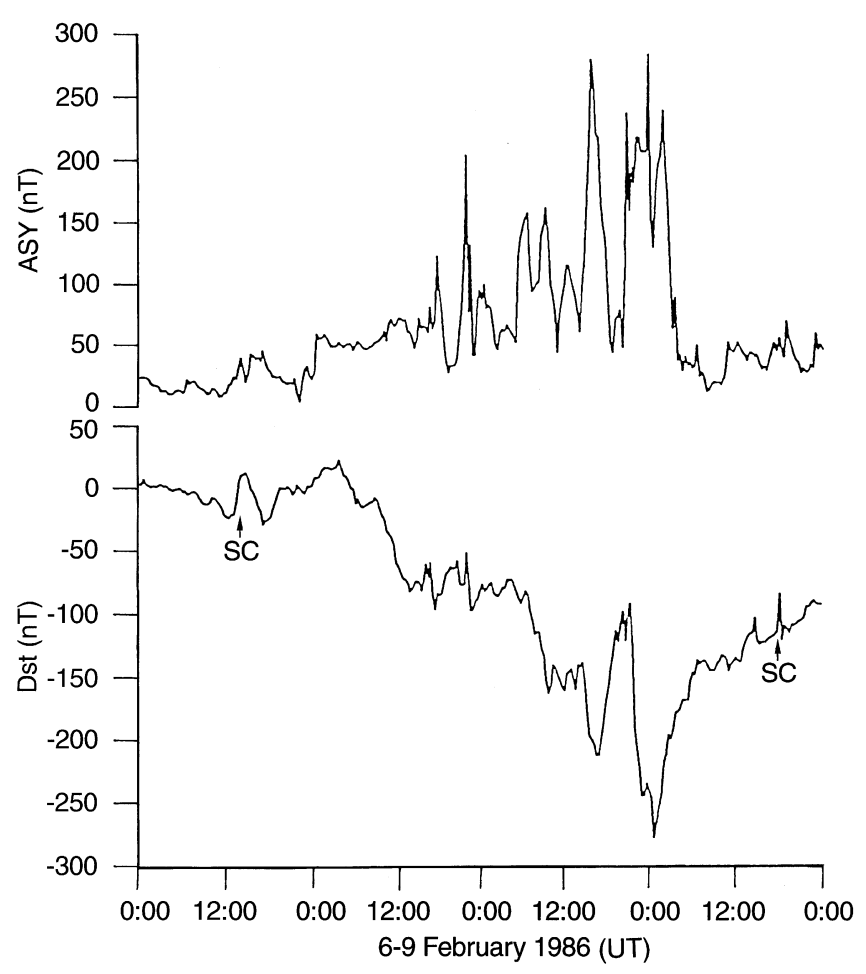

Fig. 1. Variation of Dst in low latitudes and the asymmetry of the low-latitude geomagnetic field $(A S Y)$ of the storm on February 06-09, 1986 
(1996) to estimate the evolution of the storm time magnetic field in low latitudes at all magnetic local times (MLT) was used for further analysis of this storm. This means a global picture of the ground based geomagnetic field was estimated for storm time moments (in UT). The result is depicted in Fig. 2. In principle, Fig. 2 shows a more complex geomagnetic field variation in the afternoon/evening sector than in the morning sector. During the end of the first step of the main phase at about 2100 UT on February 7 a positive variation occurs in the midnight sector and a negative one in the noon sector. The value of $A S Y$ is high at this time. Here we have an example of an anomalous $D S$ variation which is not typical for the storm main phase. The anomalous $D S$ variation was described by Grafe (1974). The cause of such a anomalous low-latitude magnetic field asymmetry is not quite clear. Does the ring current asymmetry show an anomalous behavior or increase suddenly during the main phase of the ChapmanFerraro current? Unfortunately we can not prove the latter because of missing solar wind data at this time. However, this variation can be possibly also caused by the substorm system. Indeed there is a strong substorm event occurring at about $2100 \mathrm{UT}$, as the auroral electrojet indices in Fig. 3 show. However, the question is why are no low latitude influences discernible on the strong substorms which occurred at 1015 and at 1530 UT of the same day. Therefore, I am sure that this anomalous $D S$ variation is not caused by the substorm current system.

More important for the discussion here is the evolution of the magnetic field during the second and third step of the main phase. The most active phase of the second step appears essentially in the afternoon/ evening sector. No decrease of the geomagnetic horizontal component occurs during the morning hours. The
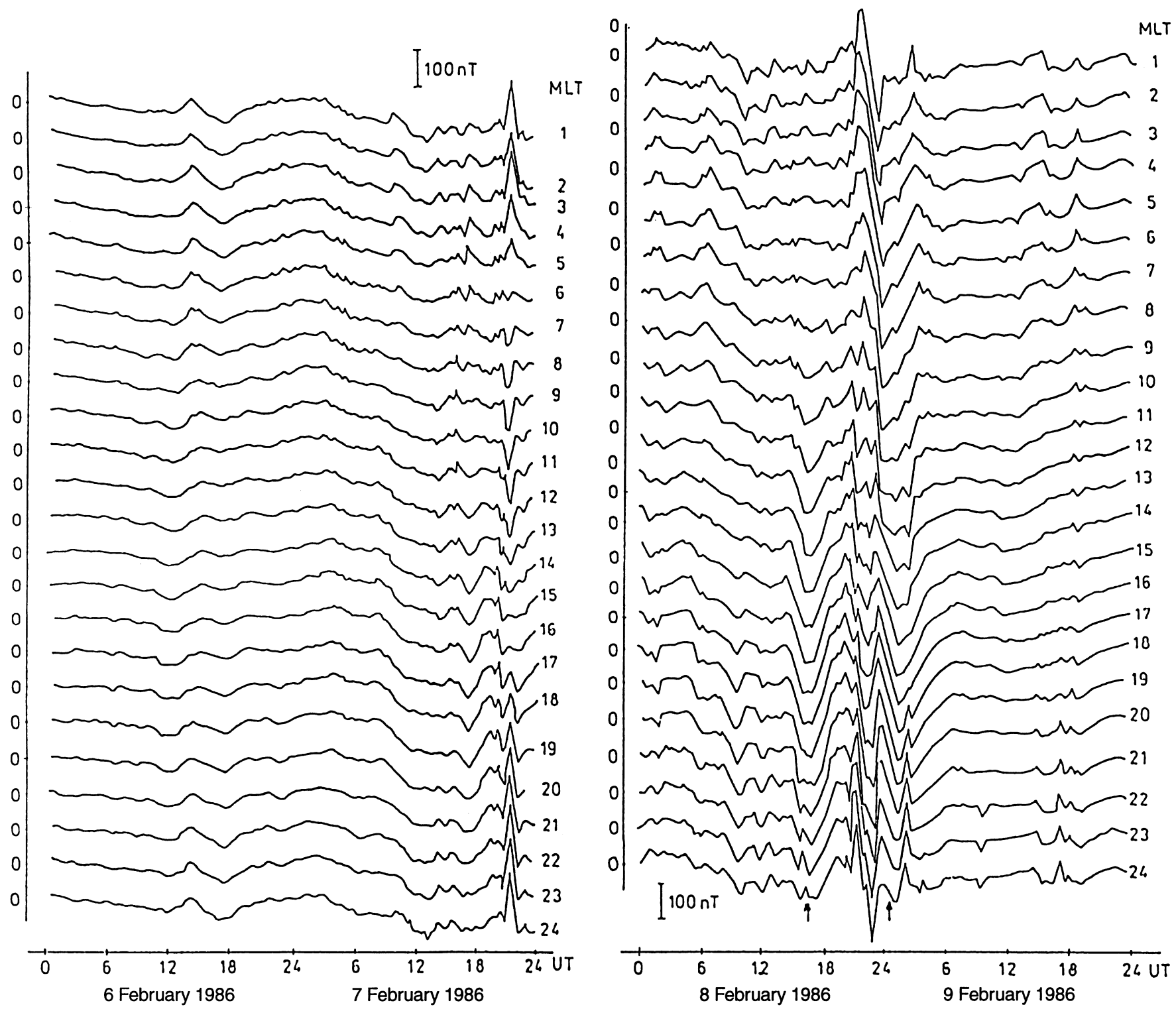

Fig. 2. Storm time variation of the geomagnetic fiel in low latitudes at different local times of the storm on February 06-09, 1986 $(\uparrow=$ beginning of the Dst recovery phase) 


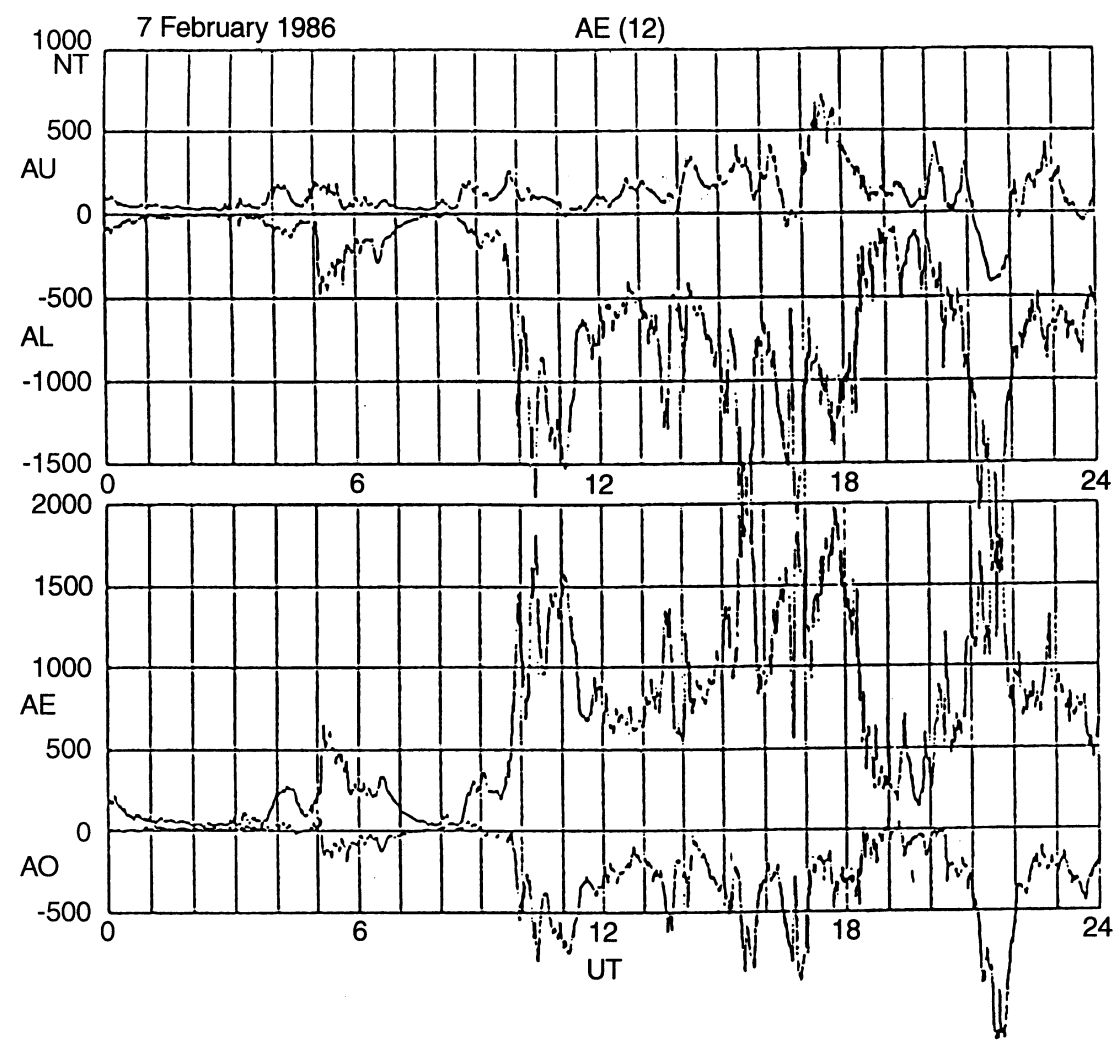

Fig. 3. Aurol electrojet indices of February 07 , 1986 third and strongest step of the main phase is characterized by magnetic field disturbances at all local times. Once again, these are most turbulent in the afternoon/ evening sector. Whereas the development in the morning sector is more or less in accordance with Dst (Fig. 1) we recognize clearly two separate decreases of the magnetic field in the evening sector (caused by consecutive increases of the ring current intensity). The arrows in Fig. 2 show the beginning of Dst recovery. We emphasize a considerable deviation at the beginning of the recovery phases in the evening sector from that in Dst. The decay of the first recovery phase is very fast in this sector. This is caused by the wave-particle interaction process found by Grafe et al. (1996). Also very remarkable is the fact that the second step of the main phase as well as the third step at first starts in the afternoon sector at about 1700/1800 MLT. Therefore, Fig. 2 supplies a very informative picture about the global development of the storm magnetic field. For instance it is logical to conclude that during the second step the energy injection of the tail was too weak to reach the whole surrounding Earth region. The injected particles were stopped in the noon sector. This also causes a high asymmetry during the recovery phase. For the third step the energy of the injection was very high. Therefore, the whole surrounding Earth region was influenced. As Fig. 2 shows, the last recovery phase of this storm ran very similarly at all MLT. This means that for strong storms the asymmetry is reduced very quickly at the beginning of the recovery phase. Considering all the informations about the global evolution of the geomagnetic storm field in Fig. 2 we should ask whether Dst supplies us with information which is correct. Concerning the beginning of the recovery phase $D s t$ provides a totally wrong picture. The recovery phase starts differently at different local times. Therefore, we have to ask whether any reasons exist to assume that in addition to an asymmetric ring current also a symmetric one acts during the storm. Keeping information from Fig. 2 in mind nothing appears to suggest this. The storm of February 06-09 is a favorable example to show that during moderately strong disturbances, contrary to strong disturbances, during a storm the asymmetry pending the recovery phase does not vanish. This was also an important result of the paper of Grafe et al. (1996). In this paper $A S Y$ was investigated during the recovery phase for storms of different intensity. The storms of moderately strong intensity do not show any disappearance of asymmetry. Figure 4 shows this fact very impressively. On the left side there are three storms of moderate intensity. For these storms the ratio of $A S Y$ (recovery phase) $/ A S Y$ (main phase) is higher than for the strong storms on the right side. However, it is very important that the asymmetry does not vanish during the recovery phase. Independent of the intensity of the storm this asymmetry has values between 50 and $100 \mathrm{nT}$.

\section{Statistical results}

The goal of this section is to find the relationships between Dst and ASY during main and recovery phase for storms of different activity. Dst and ASY from 19891995 and of some very powerful storms (September 

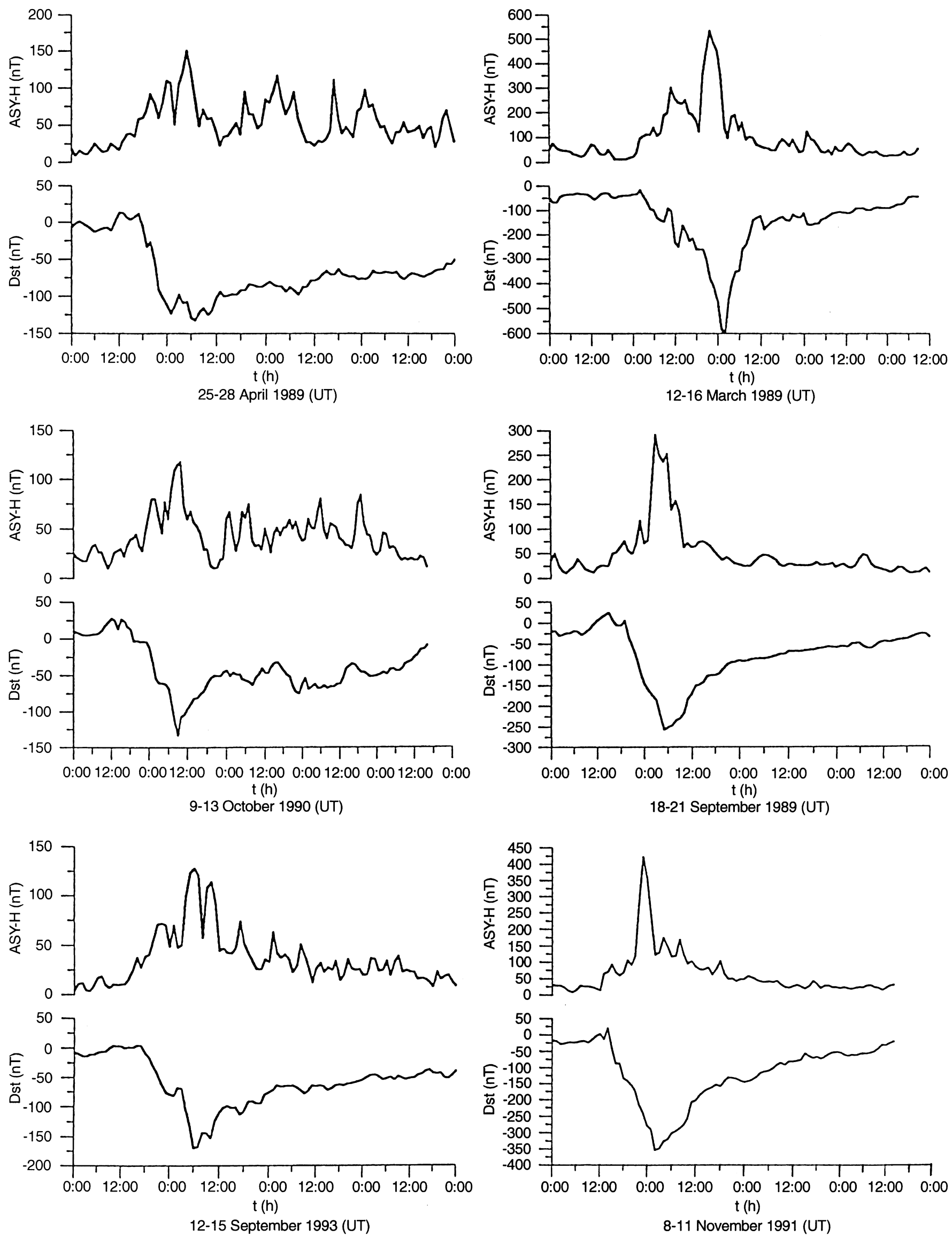

Fig. 4. Some examples of the variation of Dst and ASY for moderate (left side) and strong (right side) storms 
1957, February 1958, May 1967) were used to investigate these relationships. Figure 5 shows some of these relationships. On the left hand side the relationship between the maximum value of $|D s t|$ and maximum of $A S Y$ during the storm main phase if represented. The well-known effect that the asymmetry increases when the absolute value of $D s t$ increases is clearly shown by Fig. 5. However, because this positive correlation is caused by the very strong storms it is not linear. Taking into account a linear relationship between $|D s t(\max )|$ and $A S Y(\max )$ the asymmetry for very strong storms is lower than expected. This is depicted also on the right hand side of Fig. 5. The ratio between $A S Y(\max )$ and $D s t(\max )$ decreases with increasing $|D s t|$.

Figure 6 shows the relationship between the asymmetry during the recovery phase and the $|D s t|$ during the main phase using again the maximum value of Dst. The $A S Y$ mean value for $10 \mathrm{~h}$ after the beginning of the recovery phase was taken as a characteristic value of the asymmetry during the recovery phase. The upper representation clearly shows that the asymmetry also increases with increasing storm intensity during the recovery phase but the linearity of this relationship is violated for very strong storms. This corresponds to the conception that the storm time magnetic field symmetry is reached more or less at the end of the main phase when considering very strong storms. However for moderate storms symmetry is not reached at the end of the main phase.

The relationship between the ratio of the asymmetry during the recovery phase to maximum $|D s t|$ during the main phase and the intensity of the storm is shown on the left hand side of the lower part of Fig. 6. Notwithstanding the relatively high scattering of this ratio for weak storms it can be clearly seen that this ratio is low for very strong storms. Therefore, the asymmetry during the recovery phase is slightly higher for moderate storms than for strong storms. This statistical result is in a good accordance with the result of the special storm on February 06-09, 1986. In Fig. 6 on the right hand side of the lower part, the relationship is represented between the ratio of the asymmetry during recovery and main phase and the intensity of the storm. This ratio was higher than 0.5 for some storms, but was only $<0.3$ for the three strongest storms. However, this representation also shows that for all investigated storms the asymmetry was never 0 .

\section{Asymmetry of the low latitude geomagnetic field during quiet conditions}

There are rarely publications which describe the condition of the quiet time low-latitude geomagnetic field in connection with the ring current. However, it is certain that the ring current does not vanish completely during quiet conditions. Schmidt (1921) has assumed a "nulllevel" of the ring current which is the sum of all noneterminated ring current recovery phases. On the basis of the magnetic observations of Potsdam from 1892-1910 (1921) Schmidt found a value for the ring current nulllevel of about $160 \mathrm{nT}$. Today this null-level is called quiet-time ring current. It is generally assumed that this is a symmetric ring current. However, none of the best known literature which describes magnetospheric processes mentions the quiet-time ring current (see e.g., Carovillano et al. 1968; Akasofu and Chapman 1972; Jacobs 1991).
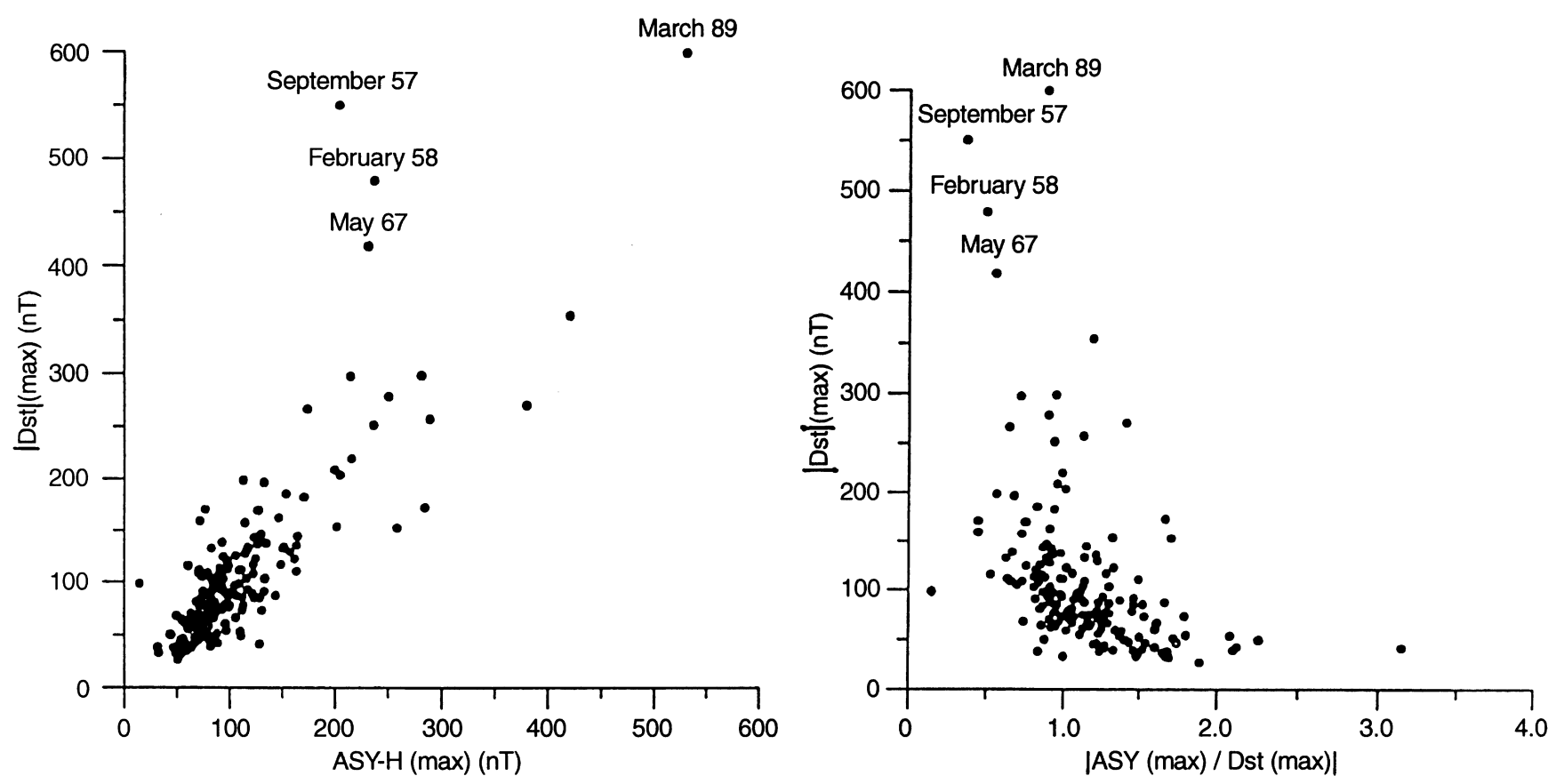

Fig. 5. Relationship between maximum $|D s t|$ and $A S Y$ and maximum $|D s t|$ and $|A S Y / D s t|$ during the storm maim phase (1989-1995 and some very strong storms) 

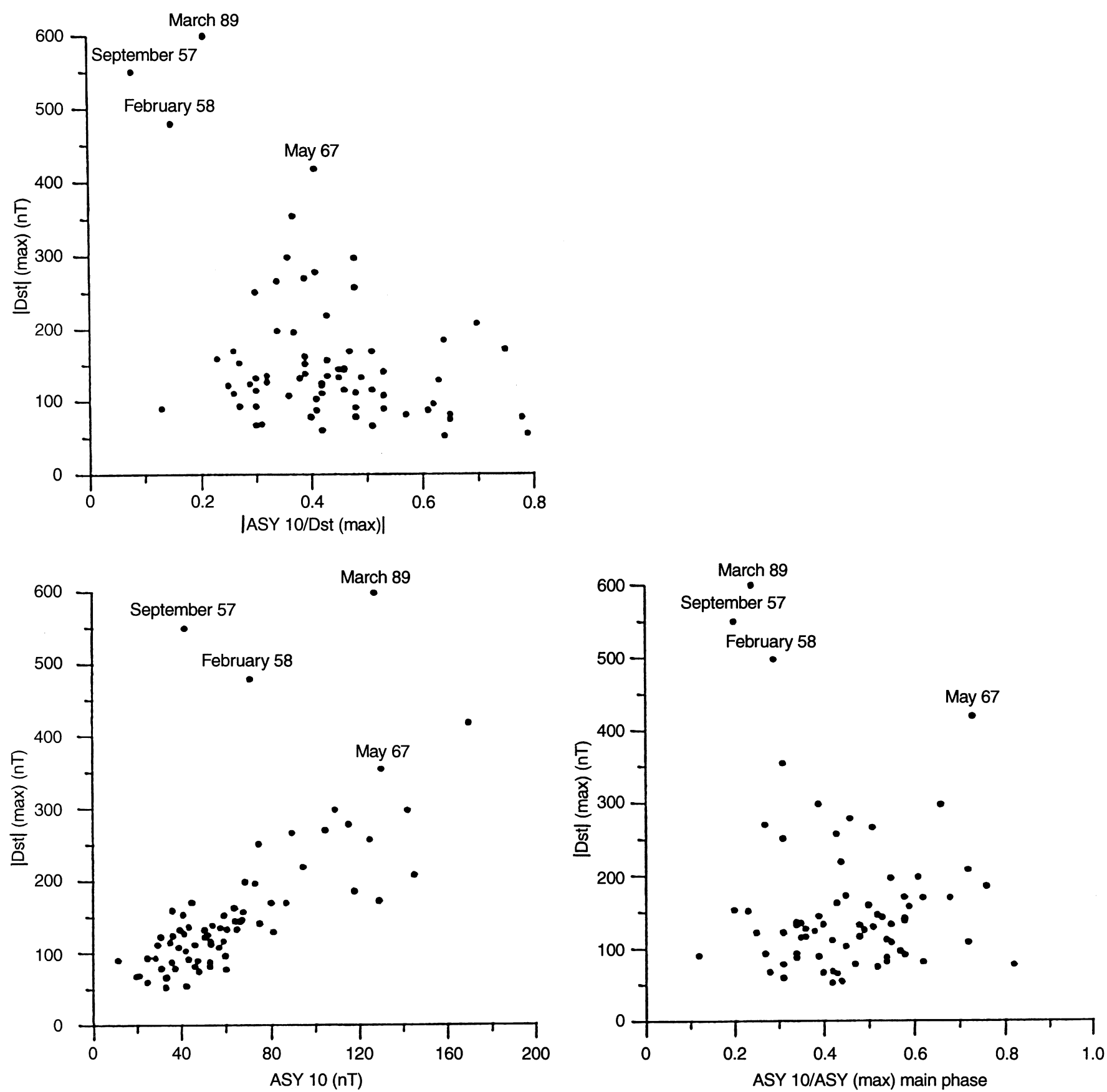

Fig. 6. Relationship between maximum $|D s t|$ and the over $10 \mathrm{~h}$ averaged values of $A S Y$ after beginning of the recovery phase (1989-1995 and some very strong storms)

The question that whether the quiet-time ring current is symmetric or asymmetric is of fundamental importance. Therefore, I shall try to answer this question, in the following part. In order to do this I have used the indices Dst, $A S Y$ and $A L$ for four international quiet days of 1990. All these indices were supplied from the World Data Center C2 for Geomagnetism of the Kyoto University. The variation of the hourly mean values of these indices is represented in Fig. 7. The observation that the field asymmetry is often greater than Dst is an essential conclusion from Fig. 7. Sometimes $A S Y$ reaches $40 \mathrm{nT}$ or more (on February 13, 1990). Also when Dst is about zero $A S Y$ can reach $30 \mathrm{nT}$ (on March 17, 1990 and October 08, 1990). This means that the low-latitude magnetic field asymmetry does not vanish during quiet conditions. As it is also shown by Fig. 7 that the asymmetry on these four quiet days cannot be caused by a substorm current system because $A L$ is very low.

However, we have to ask further: is it possible that this asymmetry can be actually caused by an asymmetric ring current or is another explanation imaginable? We can give a clear answer in Fig. 8 where local time profiles of the averaged ASY values are shown representing five international quiet days of February 1990. 

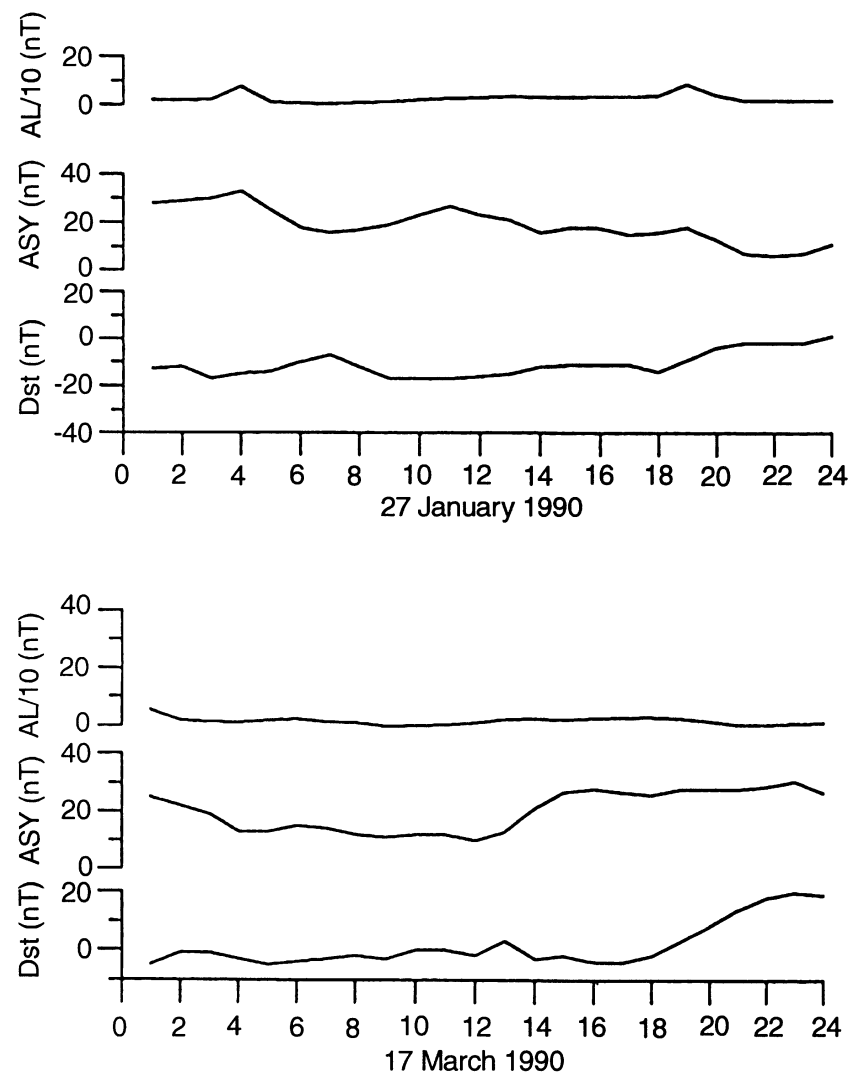

Fig. 7. Dst, $A S Y$, and $A L / 10$ for four international quiet days of 1990

These data were measured at the observatories listed in Table 2. To estimate the $D S 1$ variation the observation points were balanced using the method of least squares at all UT. The observations are in a good correspondence with the calculated $D S 1$ variation. The minimum of $D S 1$ always appears in the evening sector. This is a clear feature of an asymmetric ring current and cannot be explained by another cause. Therefore we have to assume that the quiet-time ring current is always an asymmetric one.

\section{Discussion}

The observations of the Satellite OGO3 (Frank, 1970) have shown that the observed asymmetry of the lowlatitude geomagnetic field during the storm main phase can be explained by an asymmetric distribution of the trapped ions in the inner magnetosphere. Thus the appearance of the maximum ion density was observed in the evening sector. This maximum is also in accordance with the observed maximum of the geomagnetic field decrease occurring in the same sector. Thus the existence of an asymmetric ring current during the storm main phase was established by these $\mathrm{OGO} 3$ observations. However, do any of these observations contain clear evidence entitling us to assume that there also is a symmetric ring current operating independently from
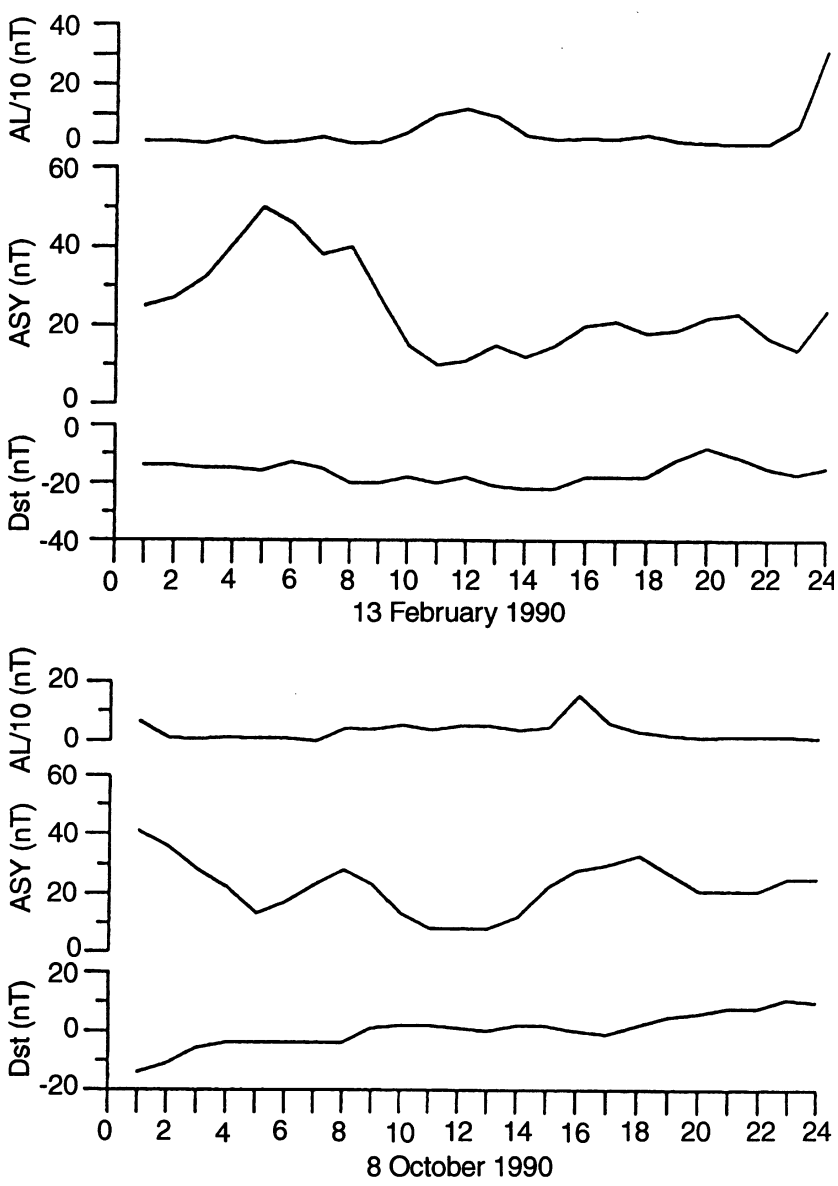

the asymmetric one? I think these observations do not allow us to conclude from $D s t$ that a symmetric ring current exists. The investigation of the ground-based low-latitude geomagnetic field has yielded following results.

1. The storm time geomagnetic field develops very differently at different local times.

2. There are differences between storms of different intensity concerning the entry of ring current particles into the Earth's environment. During strong storms the ring current energy is obviously high enough to quickly reach all regions surrounding the Earth. With weaker storms, the energy is not so high, and therefore this energy will be stopped in the evening/ afternoon sector. Moreover, the high main phase asymmetry vanishes very quickly for strong storms and reaches already typical recovery phase asymmetrics at the beginning of the recovery phase.

3 . The asymmetry during the recovery phase is never zero. The values are between 50 and $100 \mathrm{nT}$.

4. During quiet magnetospheric conditions the local time geomagnetic field asymmetry is not zero in low latitudes. It reaches an average of 30-40 nT. In some cases it can even exceed $50 \mathrm{nT}$. The minimum value of the geomagnetic horizontal component always appears in the evening sector. This is evidence that the asymmetry is caused by an asymmetric ring current. 

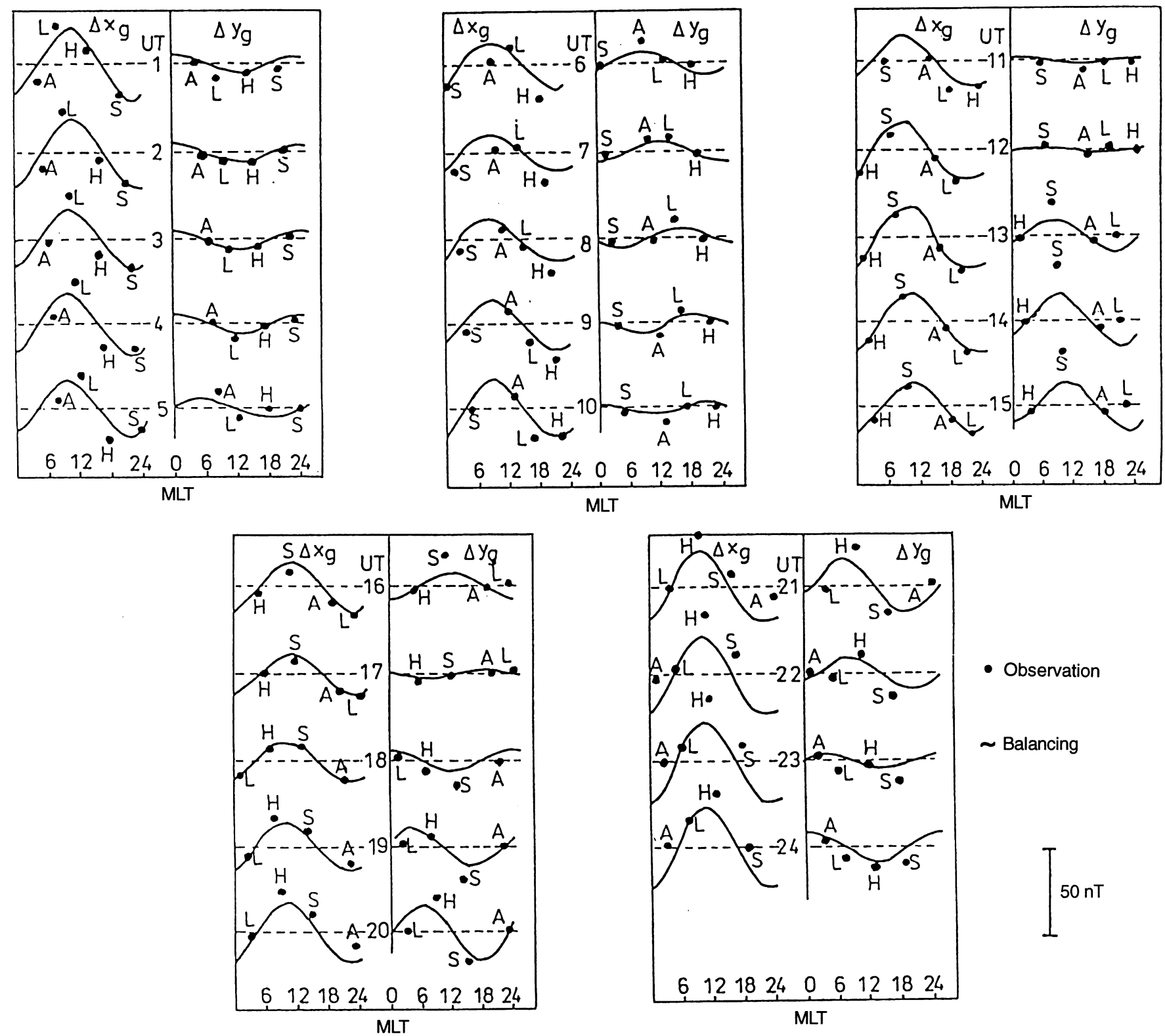

Fig. 8. Averaged local time profiles of $A S Y$ calculated from the five international quiet days of February, 1990 ( $\triangle X g$, geomagnetic north component, $\Delta Y g$, geomagnetic east component, $H$, Honolulu, $S$, San Juan, $A$, Ashchabad, $L$, Lunping)

Therefore, we conclude that the ring current is also asymmetric during quiet conditions.

Taking into account all these results I can draw the following picture: the trapped particle population in the inner magnetosphere has an asymmetric distribution in

Table 2.

\begin{tabular}{llllll}
\hline Observatories & \multicolumn{2}{l}{ Geographic } & & \multicolumn{2}{l}{ Geomagnetic } \\
\cline { 2 - 3 } \cline { 5 - 6 } \cline { 5 - 6 } & latitude & longitude & & latitude & longitude \\
\hline San Juan & 18.1 & 293.9 & & 29.4 & 4.6 \\
Ashchabad & 38.0 & 58.1 & & 30.4 & 134.3 \\
Lunping & 25.0 & 121.2 & & 13.9 & 190.7 \\
Honolulu & 21.3 & 202.0 & & 21.4 & 268.0 \\
\hline
\end{tabular}

the local time. This is also valid during a quiet magnetosphere. The effect of this asymmetric population is an asymmetric ring current. There is no evidence for a symmetric particle population and a symmetric ring current during quiet conditions. During the storm main phase the asymmetry increases and very strongly for the strongest storms. However, this strong asymmetry vanishes very quickly, and this "storm time asymmetry" has mostly disappeared by the beginning of the storm recovery phase. Again "quiet time asymmetry" conditions are reached. At no time is the ring current symmetric. Therefore, the most important conclusion I can draw from this investigation is: in contradiction to the generally valid opinion of two separated ring currents, a symmetric and an asymmetric one, in my opinion exists only an asymmetric ring current in the 
inner magnetosphere. This ring current changes its degree of asymmetry and shifts its current maximum from the inner boundary of the plasma sheet towards the Earth during disturbed conditions. However, what is then the meaning of Dst? Dst is only a mathematical parameter and does not represent an index of a symmetric ring current. Certainly, Dst has its importance as an useful index characterizing the middle course of geomagnetic storms.

Acknowledgements. Topical Editor K.-H. Glassmeie thanks W. H. Campbell and another referee for their help in evaluating this paper.

\section{References}

Akasofu, S. I., and S. Chapman, On the asymmetric development of magnetic storm fields in low and middle latitudes, Planet. Space Sci., 12, 607, 1964.

Akasofu, S.-I., and S. Chapman., Solar-terrestrial physics, 1972, Oxford University Press.

Alexeev, I. I., E. S. Belenkaya, V. V. Kalegaev, Y. I. Feldstein, and A. Grafe., Magnetic storms and magnetotail currents, J. Geophys. Res., 101, 7737, 1996.

Cahill, L. J. Jr., Inflation of the inner magnetosphere during a magnetic storm, J. Geophys. Res., 71, 4505, 1966.

Campbell, W. H., Field levels near midnight at low and equatorial geomagnetic stations, J. Atmos. Terr. Phys., 35, 1127, 1973.

Campbell, W. H., Geomagnetic storms, the Dst ring-current myth, and lognormal distributions, J. Atmos. Terr. Phys., 58, 1171, 1996.

Campbell, W. H., Introduction to geomagnetic fields, Cambridge University Press, 304 p., 1997.

Carovillano, R. L., J. F. McClay, and H. R. Radoski, Physics of the magnetosphere, D. Reidel, Dordrecht-Holland, 1968.

Chapman, S., An outline of a theory of magnetic storms, Proc. R. Soc. London. A95, 61, 1919.

Crooker, N. U., and R. L. McPherron, On the distinction between the auroral electrojet and partial ring current systems, J. Geophys. Res., 77, 6886,1972.

Cummings, W. D., Asymmetric ring currents and the low-latitude disturbance daily variation, J. Geophys. Res., 71, 4495, 1966.

Frank, L. A., On the extraterrestrial ring current during geomagnetic storms, J. Geophys. Res., 72, 3753, 1967.
Frank, L. A., Direct detection of asymmetric increases of extraterrestrial ring current proton intensities in the outer radiation zone, J. Geophys. Res., 75, 1263, 1970.

Fukushima, N., and Y. Kamide, Partial ring current models for worldwide geomagnetic disturbances, Rev. Geophys. Space Phys., 11, 795, 1973.

Fuller-Rowell, T. J., M. V. Codrescu, R. J. Moffet, and S. Quegan, Response of the thermosphere and ionosphere to geomagnetic storms, J. Geophys. Res., 99, 3893, 1994.

Grafe, A., Probleme der Analyse des Ringstromfeldes in Verbindung mit den Dst-Variationen, Gerl. Beitr. Geoph., 77, 92, 1968.

Grafe, A., Anomalous DS variation in equatorial latitudes during geomagnetic storms, Planet. Space Sci., 22, 991, 1974.

Grafe, A., V. Y. Trakhtengerts, P. A. Bespalov, and A. G. Demekhov, Evolution of the low-latitude geomagnetic storm field and the importance of turbulent diffusion for ring current particle losses, J. Geophys. Res., 101, 24689, 1996.

Jacobs, J. A., Geomagnetism, Vol. 4, Academic Press, 1991.

Kamide, Y., and N. Fukushima, Analysis of magnetic storms with DR-indices for equatorial ring current field, Rep. Ionos. Space Res. Japan, 25, 125, 1971.

Kawasaki, K., and S. -I. Akasofu, Low latitude DS component of geomagnetic storm field, J. Geophys. Res., 76, 2396, 1971.

Lui, A. T. Y., R. W. McEntire, and S. M. Krimigis, Evolution of the ring current during two geomagnetic storms, J. Geophys. Res., 92, 7459, 1987.

Moos, N. A. S., Magnetic observations made at the government observatory for the period 1846 to 1905, Part II, Bombay, 1910.

Schmit, A., Die erdmagnetische Säkularvariation, Phys. Z. 22, 152, 1921.

Siscoe, G. L., and N. U. Crooker, On the ring current contribution to Dst, J. Geophys. Res., 79, 1110, 1974.

Sugiura, M., Hourly values of equatorial Dst for the IGY, Annals $I G Y, 35$, (I), 9-45,1964.

Sun, W., B. H. Ahn, S. -I. Akasofu, and Y. Kamide, A comparison of the observed mid-latitude magnetic disturbance field and those reproduced from the high latitude modeling current system, J. Atoms. Terr. Phys., 35, 10881, 1984.

Vestine, E. H., L. Laporte, I. Lange and W. E. Scott, The geomagnetic field, its description and analysis, Carnegie Institute of Washington, Pub. 580, 1-390, 1947.

Williams, P. J. Ring current composition and sources: an update, Planet. Space Sci 29, 1195, 1981.

Yacob, A, Day-time enhancement of geomagnetic disturbances at the magnetic equator in India, J. Meteorol. Geophys., 35, 1127, 1973. 